\title{
Clusterin Induces MUC5AC Expression via Activation of NF- $\kappa$ B in Human Airway Epithelial Cells
}

\author{
Chang Hoon Bae ${ }^{1}$ Hyung Gyun $\mathrm{Na}^{1} \cdot$ Yoon Seok Choi ${ }^{1}$ Si-Youn Song ${ }^{1} \cdot$ Yong-Dae Kim,2 \\ ${ }^{1}$ Department of Otorhinolaryngology-Head and Neck Surgery, Yeungnam University College of Medicine, Daegu; \\ ${ }^{2}$ Regional Center for Respiratory Diseases, Yeungnam University Medical Center, Daegu, Korea
}

Objectives. Clusterin (CLU) is known as apolipoprotein $\mathrm{J}$, and has three isoforms with different biological functions. CLU is associated with various diseases such as Alzheimer disease, atherosclerosis, and some malignancies. Recent studies report an association of CLU with inflammation and immune response in inflammatory airway diseases. However, the effect of CLU on mucin secretion of airway epithelial cells has not yet been understood. Therefore, the effect and brief signaling pathway of CLU on MUC5AC (as a major secreted mucin) expression were investigated in human airway epithelial cells.

Methods. In the tissues of nasal polyp and normal inferior turbinate, the presence of MUC5AC and CLU was investigated using immunohistochemical stain and Western blot analysis. In mucin-producing human NCI-H292 airway epithelial cells and primary cultures of normal nasal epithelial cells, the effect and nuclear factor kappa-light-chain-enhancer of activated B cells (NF-kB) signaling pathway of CLU on MUC5AC expression were investigated using immunohistochemical stain, reverse transcription-polymerase chain reaction, real-time polymerase chain reaction, enzyme immunoassay, and Western blot analysis.

Results. In the nasal polyps, MUC5AC and CLU were abundantly present in the epithelium on immunohistochemical stain, and nuclear CLU (nCLU) was strongly detected on Western blot analysis. In human NCI-H292 airway epithelial cells or the primary cultures of normal nasal epithelial cells, recombinant nCLU increased MUC5AC expression, and significantly activated phosphorylation of NF-кB. And BAY 11-7085 (a specific NF-kB inhibitor) and knockdown

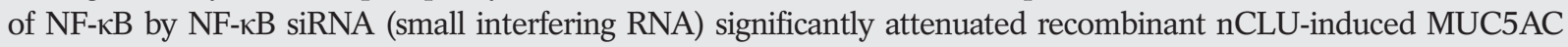
expression.

Conclusion. These results suggest that $\mathrm{nCLU}$ induces MUC5AC expression via the activation of NF-kB signaling pathway in human airway epithelial cells.

Keywords. Clusterin; Nasal Polyps; MUC5AC; NF-kappa B; Epithelial Cells

\section{INTRODUCTION}

Chronic rhinosinusitis (CRS) is a broad clinical syndrome that is defined by mucosal inflammation of the nose and paranasal sinuses, which is subdivided into two groups based the presence

\footnotetext{
- Received May 2, 2017

Revised October 8, 2017

Accepted November 10, 2017

- Corresponding author: Yong-Dae Kim

Department of Otorhinolaryngology-Head and Neck Surgery, Yeungnam

University College of Medicine, 170 Hyeonchung-ro, Nam-gu, Daegu

42415, Korea

Tel: +82-53-620-3781, Fax: +82-53-628-7884

E-mail: ydkim@med.yu.ac.kr
}

or absence of nasal polyps (NP). Among them, chronic rhinosinusitis with nasal polyps (CRSwNP) is one of the most difficult upper airway diseases to treat $[1,2]$. In CRSwNP, mucus hypersecretion is major symptom, which is associated with goblet cell metaplasia and hypertrophy of nasal airway epithelium. Mucins are high molecular weight glycoproteins, one of major components responsible for mucus secretion, impart different physical and biological properties to mucus of the airway $[3,4]$. Among the mucins, MUC5AC is suggested to be a main marker of mucous metaplasia in sinonasal mucosa and predominant expressed in CRSwNP patients [2]. Nevertheless, the mechanisms underlying overproduction of MUC5AC glycoprotein in CRSwNP are not fully understood.

Copyright @ 2018 by Korean Society of Otorhinolaryngology-Head and Neck Surgery

This is an open-access article distributed under the terms of the Creative Commons Attribution Non-Commercial License (http://creativecommons.org/licenses/by-nc/4.0)

which permits unrestricted non-commercial use, distribution, and reproduction in any medium, provided the original work is properly cited. 
Clusterin (CLU), a heterodimeric disulfide-linked glycoprotein, is known as apolipoprotein $\mathrm{J}$, and has three active isoforms with distinct subcellular locations and different biological functions: presecretory CLU (psCLU), secretory CLU (sCLU), and nuclear CLU (nCLU). These isoforms are abundantly and widely distributed in human tissues. psCLU and sCLU are thought to be correlated with cytoprotective and anti-inflammatory reactions in various organs. Meanwhile, nCLU is known to be responsible for promoting cell apoptosis [5]. CLU is associated with cardiovascular disease, neurodegenerative disorders, cancer progression, metabolic disease, and respiratory disease [6,7]. In recent studies on the association of CLU with respiratory diseases, CLU levels of serum and sputum from asthmatic patients clearly correlated with increased oxidative stress status $[8,9]$. And CLU is also strongly expressed in the airway submucosa of smokers and has been found to protect airway fibroblasts from oxidative stress [10]. Moreover, CLU is reported to exhibit intraand extracellular interactions with inflammation-associated molecules, such as complement factors, nuclear factor of kappa light polypeptide gene enhancer in B-cells inhibitor $\alpha$, and transforming growth factor $\beta$, suggesting that it plays an important role in modulating conditions related to inflammation and immune responses [11-13]. Through these previous studies, the authors hypothesized that CLU would be closely related to mucus hypersecretion, one of the major causes of development and persistence of inflammatory airway diseases.

Therefore, we investigated whether which isoforms of CLU is expressed in nasal epithelium of CRSwNP patients, and the expression level of CLU is different in CRSwNP patients in comparison with normal nasal epithelium. And we evaluated the effect and involved regulatory mechanism of CLU on the expression of MUC5AC (as a marker of mucus hypersecretion) in human airway epithelial cells.

\section{MATERIALS AND METHODS}

This study was approved by the Institutional Review Board for human studies at Yeungnam University Medical Center (IRB No. YUMC 2015-06-059) and written informed consent was obtained from each patient.

\section{H I G H L I G H H T S}

- Clusterin induces MUC5AC expression.

- Clusterin was abundantly present in the epithelium of nasal polyp.

- Clusterin induces MUC5AC expression via the activation of nuclear factor kappa-light-chain-enhancer of activated B cells (NF-kB).

\section{Materials}

Human recombinant nCLU was obtained from AdipoGen (Incheon, Korea). Mucin-producing human NCI-H292 airway epithelial cells were obtained from the American Type Culture Collection (Manassas, VA, USA). RPMI 1640 medium was obtained from Invitrogen (Carlsbad, CA, USA). EpiLife medium and human keratinocyte growth supplement were obtained from Cascade Biologics (Portland, OR, USA). EZ-Cytox cell viability assay kit was obtained from Daeil Lab (Seoul, Korea). The specific inhibitor, BAY 11-7085 was obtained from SigmaAldrich (St. Louis, MO, USA). Reverse transcription-polymerase chain reaction (RT-PCR) kits were obtained from Applied Biosystems (Foster City, CA, USA). Real-time PCR kits were obtained from Roche Applied Science (Mannheim, Germany). For immunohistochemical stain, primary antibody of MUC5AC, secondary antibody, and streptavidin-horseradish peroxidase, and 3,3'-diaminobenzidine substrate were obtained from $\mathrm{Ab}$ cam (Cambridge, UK). Primary antibody of CLU was obtained from Santa Cruz Biotechnology (Santa Cruz, CA, USA). For immunoassay analysis, primary and secondary antibody of MUC5AC were obtained from Santa Cruz Biotechnology. For Western blot analysis, primary and secondary antibody of CLU, and nuclear factor kappa-light-chain-enhancer of activated B cells (NF- $\mathrm{KB}$ ) were purchased from Cell Signaling Technology (Danvers, MA, USA). Enhanced chemiluminescence system kits were obtained from PerkinElmer Life Sciences (Boston, MA, USA). For cell transfection with siRNA (small interfering RNA), predesigned siRNA targeting NF-kB, control siRNA, Opti-MEM I reduced serum medium, and Lipofectamine 2000 were obtained from Invitrogen.

\section{Tissue collection}

Nasal polyp tissues originated from middle turbinate were obtained from patients suffering with CRSwNP during endoscopic sinus surgery (five males and five females; mean age, 45.6 years). Nasal mucosa tissues of normal inferior turbinate were obtained from 10 patients undergoing augmentation rhinoplasty and both inferior turbinoplasty for correction of cosmetic and functional (nasal obstruction) problems (five males and five females; mean age, 35.6 years). They had no personal or family history of allergy and negative results on both allergy specific tests (skin-prick test and multiple allergen simultaneous test).

\section{Cell culture and treatment}

Human NCI-H292 airway epithelial cells were cultured in RPMI 1640 medium supplemented with $2 \mathrm{mM}$ L-glutamine, $100 \mathrm{U} /$ $\mathrm{mL}$ penicillin, $100 \mu \mathrm{g} / \mathrm{mL}$ streptomycin, and $10 \%$ fetal bovine serum. The cells were grown at $37^{\circ} \mathrm{C}$ in $5 \% \mathrm{CO}_{2}$ fully humidified air and subcultured twice weekly. When the cultures were confluent, the cells were incubated in RPMI 1640 medium containing $0.5 \%$ fetal bovine serum for 24 hours. The cells were then rinsed with serum-free RPMI 1640 medium and exposed to the 
indicated concentrations of recombinant nCLU.

For the primary culture of human nasal epithelial cells, the nasal mucosal tissue was washed with phosphate-buffered saline (PBS) and immersed in dispase for 90 minutes. After the tissue was scraped off the surface of the nasal mucosa using a scalpel, it was added to $1 \%$ PBS and filtered through a mesh. The cells were seeded in a 24 -well plate at $2.5 \times 10^{5}$ cells/well and incubated with EpiLife medium and human keratinocyte growth supplement ( $5 \mathrm{~mL} / 500 \mathrm{~mL}$ of medium). When the cultures were confluent, the cells were exposed to the indicated concentrations of recombinant nCLU.To compare recombinant nCLU exposed-cells and nCLU-unexposed-cells, NCI-H292 cells and primary culture of human nasal epithelial cells from normal inferior turbinate mucosa were incubated with the medium alone as a control. The cell proliferation and viability of NCI-H292 cells and primary culture of normal nasal epithelial cells were quantified using an EZ-Cytox cell viability assay kit, and the changes of cell morphology were examined using a microscope.

\section{Immunohistochemical stain for MUC5AC and CLU}

Immunohistochemical analysis was performed on $6 \mu \mathrm{M}$ thick sections from paraffin-embedded tissues of NP and normal inferior turbinate. Sections were deparaffinized and rehydrated through xylene and alcohol. For immunoperoxidase labeling, endogenous peroxidase was blocked in $3 \% \mathrm{H}_{2} \mathrm{O}_{2}$ in methanol for 30 minutes and antigenic epitope retrieval was performed using citrate buffer, $\mathrm{pH}$ 6.0. We used the streptavidin-biotin method. CLU was detected using an unconjugated primary antibody CLU $(\alpha / \beta$ rabbit poly clonal antibody H330, sc-8354; Santa Cruz Biotechnology), which recognizes all CLU isoforms. The CLU antibody was used at 1:200 di lution for overnight incubation at $4^{\circ} \mathrm{C}$. And we used goat anti-rabbit biotinylated secondary antibody (Abcam). After washing in PBS, we added 100 $\mu \mathrm{L}$ streptavidin-horseradish peroxidase conjugates to the sections in the slides and incubated in a humidified chamber at room temperature for 30 minutes in the dark. Horseradish peroxidase activity was visualized by $3,3^{\prime}$-diaminobenzidine substrate solution. For the negative control, nonimmune serum immunoglobulin $\mathrm{G}$ was used instead of primary antibody. The slides were counterstained with hematoxylin and mounted. The same procedure was performed with MUC5AC.

Immunohistochemical stains of MUC5AC and CLU were analyzed on the three different sections in five tissues per group and classified by two independent, blinded observers using a semi-quantitative method. Intensity of staining was scored as 0 , negative; $1+$, weak; $2+$, moderate; $3+$, strong. Extend of stained was scored as 0 , no signal in any cell; $1+$, positive signal in $<10 \%$ of cells; $2+, 10 \%-24 \% ; 3+, 25 \%-75 \%$; and $4+,>75 \%$, indicating the percentage of positive stained cells. Multiplication of intensity score (0-3) and extend score (0-4) resulted in the immunoreactivity score, which ranges between 0 and 12 [14].

\section{RT-PCR and real-time PCR analysis for MUC5AC mRNA} expression

Total RNA was isolated from human NCI-H292 airway epithelial cells and human nasal epithelial cells according to the manufacturer's instructions (Applied Biosystems). Each sample was reverse transcribed into cDNA using the GeneAmp RNA PCR core kit (Thermo Fisher Scientific, Waltham, MA, USA). PCR for glyceraldehyde-3-phosphate dehydrogenase (GAPDH) was performed on each individual sample as a positive control. Realtime PCR was performed using the LightCycler Fast Start DNA Master SYBR Green kit (Roche, Basel, Switzerland). Data were normalized to GAPDH. The primer sequences, conditions, and expression of the results followed published protocols for MUC $5 \mathrm{AC}[15,16]$.

\section{Immunoassay analysis for MUC5AC protein}

NCI-H292 cells and human nasal epithelial cells were prepared and treated with recombinant nCLU as described above. Following recombinant $\mathrm{nCLU}$ incubation for 24 hours, MUC5AC protein level was determined using an enzyme-linked immunosorbent assay (ELISA). The color was developed using 3,3', 5,5'-tetramethylbenzidine peroxidase solution and stopped with $2 \mathrm{~N}-\mathrm{H}_{2} \mathrm{SO}_{4}$. Optical density measurements were performed using an EL800 ELISA reader (BioTek Instruments, Winooski, VT, USA) at $450 \mathrm{~nm}$. The results were expressed as percent of baseline control.

Western blot analysis for $\mathrm{nCLU}, \mathrm{sCLU}$, and NF-kB

Human NCI-H292 airway epithelial cells were seeded in each well of a 6-well plate, followed by treatment to the indicated concentrations of recombinant nCLU. The cells were washed with cold PBS, exposed to trypsin, and formed into pellets at $700 \times \mathrm{g}$ for 10 minutes at $4^{\circ} \mathrm{C}$. The pellets were resuspended in lysis buffer. The proteins were separated using $10 \%$ reducing sodium dodecyl sulfate-polyacrylamide gel electrophoresis and electroblotted onto a nitrocellulose membrane. The membrane was blocked with $5 \%$ nonfat dry milk, followed by incubation with the indicated primary antibody of NF-kB for 4 hours. The membrane was incubated for 1 hour with secondary antibody of NF- $\mathrm{KB}$ conjugated to HRP, and developed using an enhanced chemiluminescence system kits. Bands were detected after exposure to X-ray film for 10 seconds. For detection of nuclear and sCLU in the NP and the normal inferior turbinate, the same procedure was performed after the tissues were homogenized.

\section{Cell transfection with siRNA for NF-kB}

To verify activation of NF- $\mathrm{KB}$ in recombinant $\mathrm{nCLU}$-induced MUC5AC expression, human NCI-H292 airway epithelial cells were transfected with a non-targeting control siRNA or NF-кB siRNA. Sequences of each siRNA and conditions of transfection were used according to the manufacturer's protocol (Invitrogen) and published protocols [16]. The transfection rate of NF- $\mathrm{kB}$ 
siRNA was verified to be over $90 \%$ in the cells. The same procedure was performed with control siRNA.

\section{Statistical analysis}

Statistical analysis was performed using SPSS ver. 12.0 (SPSS Inc., Chicago, IL, USA). The mean for each of the obtained quantitative values was calculated. Data comparisons were done with the unpaired $t$-test or Kruskal-Wallis one-way analysis of variance followed by the Mann-Whitney test. For all tests, $P$-value $<0.05$ was considered statistically significant.

\section{RESULTS}

The present of MUC5AC and CLU in the normal inferior turbinate and the nasal poly

To investigate the tissue distribution of MUC5AC and CLU, immunohistochemical stain was performed. Positive immunostained cells appeared brown. In the normal inferior turbinate, the stain for immunoreactivity of MUC5AC and CLU were present in the epithelium (Fig. 1A, B). In the NP, the stain for immunoreactivity of MUC5AC and CLU were abundantly pres- ent in the epithelium, compared to the normal inferior turbinate (Fig. 1C, D). In semi-quantitative histological analysis comparing the normal inferior turbinate and the NP, immunoreactivity scores of MUC5AC and CLU in the NP were significantly higher than those in normal interior turbinate (Fig. 1E).

To investigate the expression of CLU and its isoforms, the specific antibody to CLU was used on Western blot. The sCLU (alpha chain, $40 \mathrm{kDa}$ ) and $\mathrm{nCLU}$ (50 kDa) constitutively found in human nasal epithelium. The expression level of the nCLU is higher than that of sCLU in the normal inferior turbinate and the NP. However, the level of nCLU was strongly detected in the NP, compared to the normal inferior turbinate. On the other hand, there was no difference in the expression level of sCLU between the two groups (Fig. 1F).

\section{The effect of $\mathrm{nCLU}$ on MUC5AC expression in human $\mathrm{NCl}-\mathrm{H} 292$ airway epithelial cells}

To investigate the effect of recombinant nCLU on MUC5AC expression with dose- and time-dependent experiments, NCIH292 airway epithelial cells were treated with different doses of recombinant nCLU for 8 hours or recombinant nCLU $(5 \mu \mathrm{g}$ / $\mathrm{mL}$ ) for variable times. Results of real-time PCR and ELISA

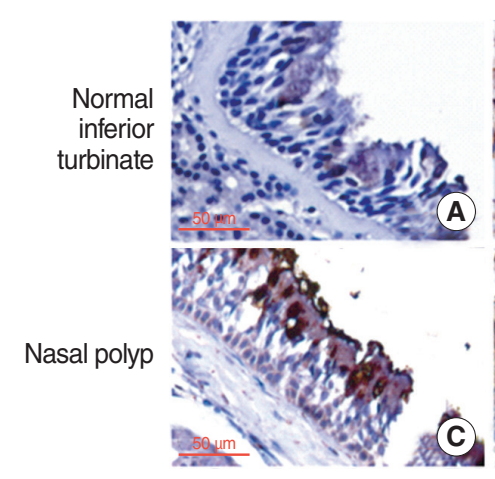

MUC5AC

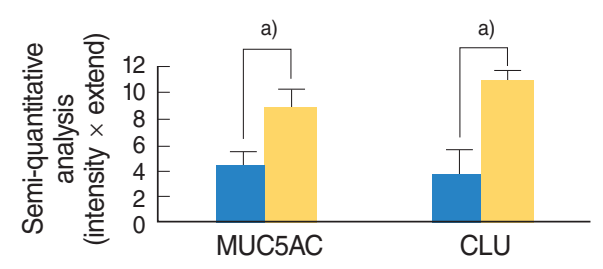

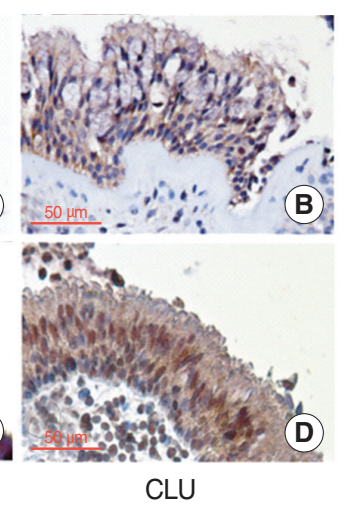

Normal inferior turbinate Nasal polyp

E
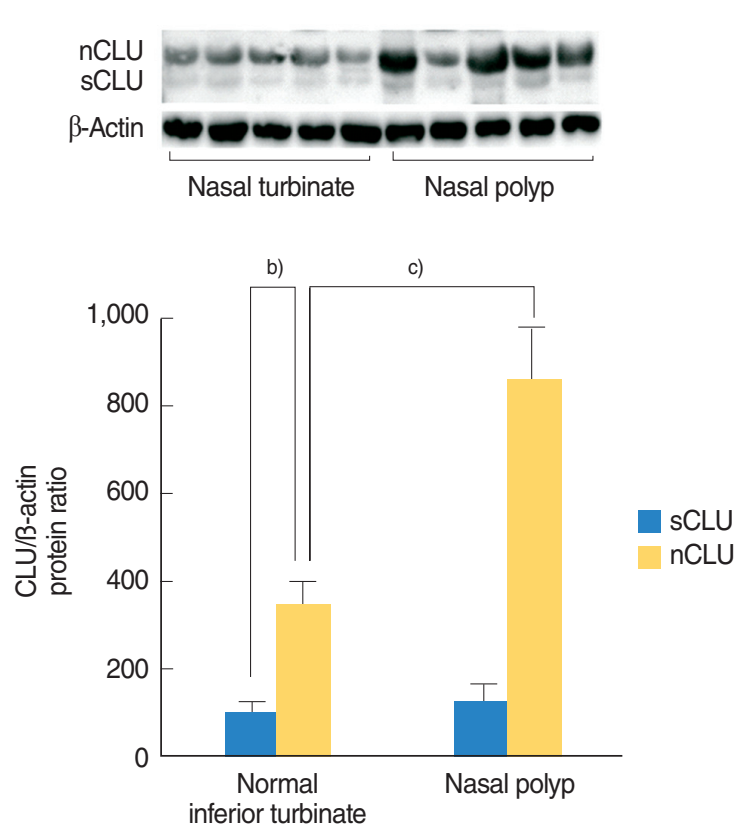

Fig. 1. The presence of MUC5AC and clusterin (CLU) in the normal inferior turbinate and the nasal polyp. (A, B) Results of immunohistochemical stain showed that MUC5AC and CLU were present in the epithelium of the normal inferior turbinate. (C, D) Results of immunohistochemical stain showed that MUC5AC and CLU were abundantly present in the epithelium of the nasal polyp. (E) Results of semi-quantitative histological analysis, immunoreactivity scores of MUC5AC and CLU in the nasal polyp were significantly higher than those in normal interior turbinate. (F) Results of Western blot showed that the secretory CLU (sCLU; alpha chain, $40 \mathrm{kDa}$ ) and nuclear CLU (nCLU; $50 \mathrm{kDa}$ ) constitutively found in human nasal epithelium. The expression level of the nCLU is higher than that of sCLU. And the level of nCLU of chronic rhinosinusitis with nasal polyps group was higher than that of healthy control. On the other hand, there was no difference in the expression levels of sCLU between the two groups. Immunopositive cells appeared brown (original magnification, $\times 400$ ). Images are representative of three separate experiments performed in triplicate. Bars indicate the average \pm standard deviation of three independent experiments performed in triplicate. ${ }^{a} P<0.05$ compared with normal inferior turbinate group. ${ }^{b)} P<0.05$ compared with sCLU from normal inferior turbinate. ${ }^{c)} P<0.05$ compared with nCLU from normal interior turbinate. 

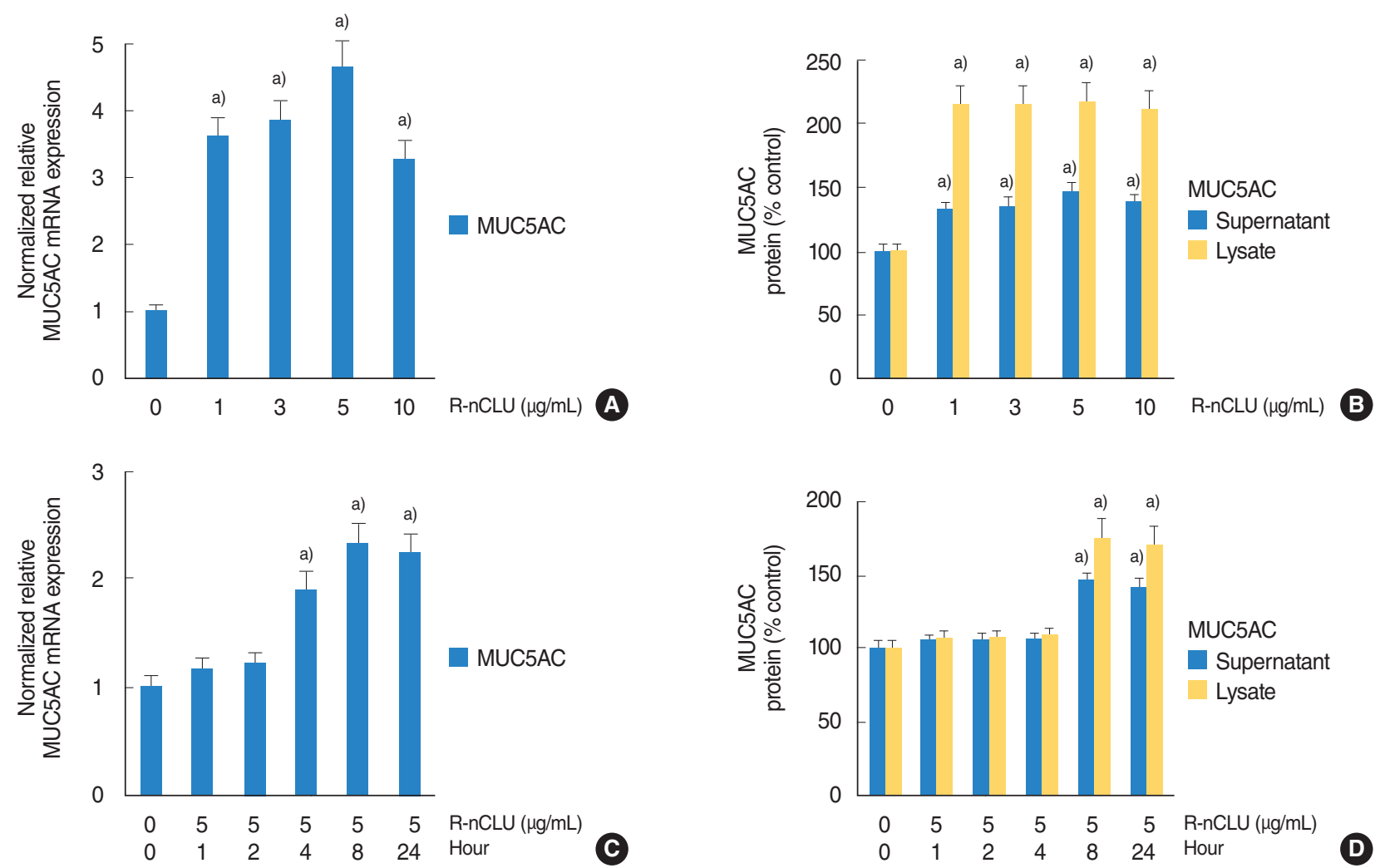

Fig. 2. The effect of nuclear clusterin (nCLU) on MUC5AC expression in human NCl-H292 airway epithelial cells. (A, B) Results of real-time polymerase chain reaction (PCR) and enzyme-linked immunosorbent assay (ELISA) showed that MUC5AC mRNA expression and protein production were significantly increased at all concentrations of recombinant nCLU (R-nCLU), and peaked at $5 \mu \mathrm{g} / \mathrm{mL}$ of R-nCLU regardless of dose-dependent manner. (C, D) Results of real-time PCR and ELISA showed that R-nCLU significantly increased MUC5AC mRNA expression and protein production regardless of time-dependent manner; MUC5AC mRNA expression and protein production were peaked at 8 hours after exposure to R-nCLU. Images are representative of three separate experiments performed in triplicate. Bars indicate the average \pm standard deviation of three independent experiments performed in triplicate. ${ }^{\text {a) }} P<0.05$ compared with zero value.

showed that MUC5AC mRNA expression and protein production were significantly increased at all concentrations of recombinant nCLU, and peaked at $5 \mu \mathrm{g} / \mathrm{mL}$ of recombinant nCLU regardless of dose-dependent manner (Fig. 2A, B). Recombinant nCLU significantly increased MUC5AC mRNA expression and protein production regardless of time-dependent manner; MUC5AC mRNA expression and protein production were peaked at 8 hours after exposure to recombinant nCLU (Fig. 2C, D).

The role of NF-kB on nCLU-induced MUC5AC expression in human $\mathrm{NCl}-\mathrm{H} 292$ airway epithelial cells

To investigate the NF-kB signaling pathway involved in nCLUinduced MUC5AC expression, human NCI-H292 airway epithelial cells were stimulated with recombinant nCLU $(5 \mu \mathrm{g} / \mathrm{mL})$, and Western blot was performed for analysis of phosphorylation of NF-kB. The phosphorylation of NF-kB was significantly increased at 60 and 120 minutes after treatment with recombinant nCLU (Fig. 3A). In addition, the cells were incubated with recombinant $\mathrm{nCLU}(5 \mu \mathrm{g} / \mathrm{mL})$ for 8 hours after pretreatment with a specific inhibitor for NF-kB (BAY 11-7085, $10 \mu \mathrm{M})$ for 1 hour. Results of RT-PCR and ELISA showed that BAY 11-7085 significantly attenuated recombinant nCLU-induced MUC5AC mRNA expression and protein production (Fig. 3B, C). To confirm this signaling pathway, the cells were transfected with NF$\kappa B$ siRNA and exposed to recombinant $\mathrm{nCLU}(5 \mu \mathrm{g} / \mathrm{mL})$. Results of RT-PCR showed that the knockdown of NF-kB by NF$\kappa B$ siRNA significantly blocked recombinant $n C L U$-induced MUC5AC mRNA expression (Fig. 3D).

\section{The effect of $\mathrm{nCLU}$ on MUC5AC expression in human nasal epithelial cells}

To investigate the effect of nCLU on MUC5AC expression in primary cultures of normal nasal epithelial cells, the cells were stimulated with recombinant nCLU $(5 \mu \mathrm{g} / \mathrm{mL})$. Results of RTPCR and ELISA showed that recombinant nCLU significantly increased MUC5AC mRNA expression and protein production (Fig. 4A, B). In addition, the cells were incubated with recombinant nCLU $(5 \mu \mathrm{g} / \mathrm{mL})$ for 8 hours after pretreatment with specific inhibitor for NF-kB (BAY 11-7085, $10 \mu \mathrm{M}$ ) for 1 hour. Results of RT-PCR and ELISA showed that BAY 11-7085 signifi- 

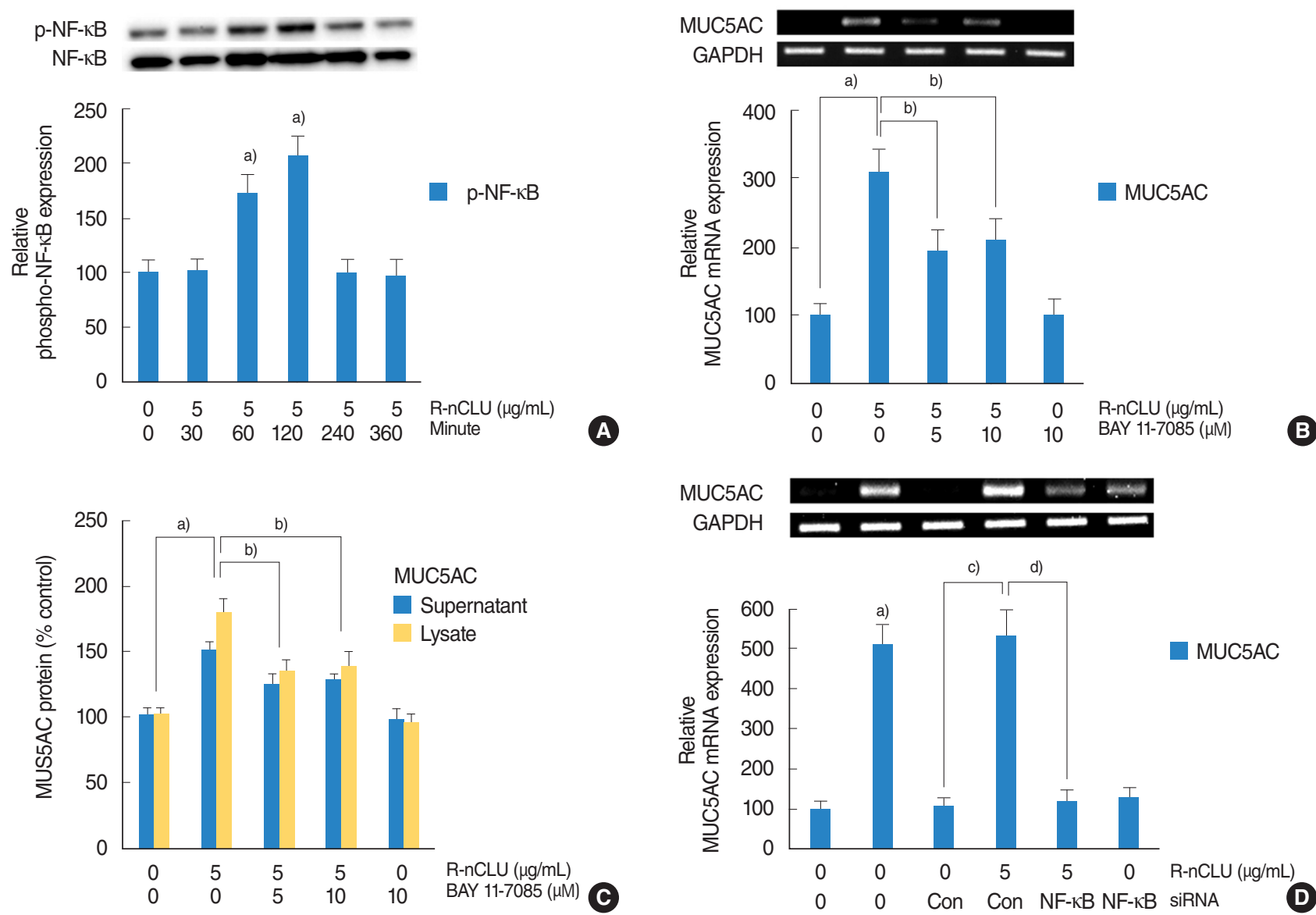

Fig. 3. The activation of nuclear factor kappa-light-chain-enhancer of activated B cells (NF-kB) on nuclear clusterin (nCLU)-induced MUC5AC expression in human NCl-H292 airway epithelial cells. (A) Results of Western blot showed that the phosphorylation of NF-kB was significantly increased at 60 minutes and 120 minutes after treatment with recombinant nCLU (R-nCLU). (B, C) Results of reverse transcription-polymerase chain reaction (RT-PCR) and enzyme-linked immunosorbent assay (ELISA) showed that BAY 11-7085 significantly attenuated R-nCLU-induced MUC5AC mRNA expression and protein production. (D) Results of RT-PCR showed that the knockdown of NF-kB by NF-kB siRNA (small interfering RNA) significantly blocked R-nCLU-induced MUC5AC mRNA expression. Images are representative of three separate experiments performed in triplicate. Bars indicate the average \pm standard deviation of three independent experiments performed in triplicate.

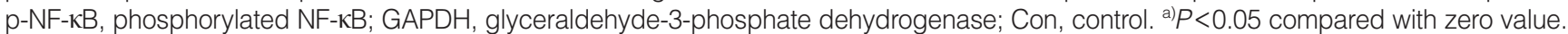
b) $P<0.05$ compared with R-nCLU alone. ${ }^{c)} P<0.05$ compared with control siRNA. ${ }^{\text {d) }} P<0.05$ compared with R-nCLU and control siRNA.

cantly attenuated recombinant nCLU-induced MUC5AC mRNA expression and protein production (Fig. 4C, D).

\section{DISCUSSION}

Mucus hypersecretion is major symptom in CRSwNP patients. And hyperplasia of mucous glands and increase in goblet cells are common histological changes in NP [1,2]. Mucins are major component responsible for mucus secretion. And overproduction of airway mucins is an important feature, which leads to an increase in the morbidity and mortality of inflammatory respiratory diseases $[3,4]$. In the studies on mucin gene expression in NP, MUC5AC overexpression was observed in sinonasal mucosa of CRSwNP and MUC5AC was expressed 4-fold higher than
MUC2 and 12-fold higher than MUC1 in NP [2,17]. The excessive production of MUC5AC was well known to mediated by many inflammatory mediators, cytokines, and hormones [16,1820]. In present study, the results of immunohistochemical stain showed that the expression level of nCLU of CRSwNP group was higher than that of healthy control group similar to the difference in the expression of MUC5AC between two groups. Therefore, we hypothesized that nCLU may play a role in MUC5AC expression in human airway epithelial cells.

CLU is a ubiquitous glycoprotein that shows a very broad pattern of tissue expression including prostate, testis, brain, liver, pancreas, lung, heart, and kidney in human [5,6,21]. The physiological functions of CLU are related to tumorigenesis, cell-substrate interaction, lipid transport, sperm maturation, tissue remodeling, cytoprotection, apoptotic cell death, cell motility, 

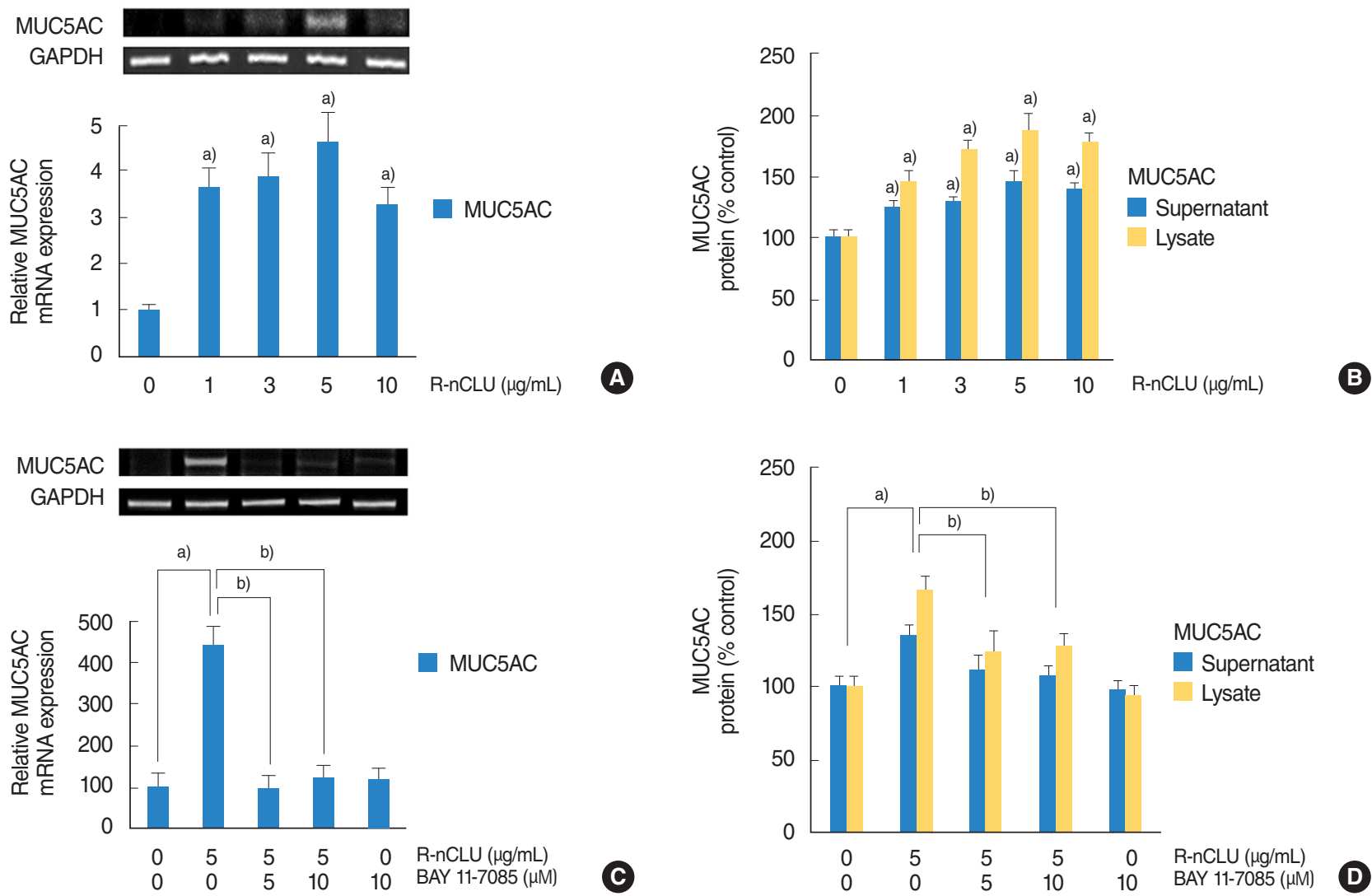

Fig. 4. The effect of nuclear clusterin (nCLU) on MUC5AC expression in human nasal epithelial cells. (A, B) Results of reverse transcriptionpolymerase chain reaction (RT-PCR) and enzyme-linked immunosorbent assay (ELISA) showed that recombinant nCLU (R-nCLU) significantly increased MUC5AC mRNA expression and protein production. (C, D) Results of RT-PCR and ELISA showed that BAY 11-7085 significantly attenuated R-nCLU-induced MUC5AC mRNA expression and protein production. Images are representative of three separate experiments performed in triplicate. Bars indicate the average \pm standard deviation of three independent experiments performed in triplicate. GAPDH, glyceraldehyde-3-phosphate dehydrogenase. ${ }^{\text {a) }} P<0.05$ compared with zero value. ${ }^{\text {b) }} P<0.05$ compared with recombinant nCLU alone.

DNA repair, and cell cycle regulation [21-23]. The CLU gene consists of nine exons from a single copy located at chromosome $8,8 \mathrm{p}$ and spans about 16.5 kilobases [6]. CLU has three active isoforms: psCLU (predicted molecular mass of $60 \mathrm{kDa}$ ), sCLU ( $80 \mathrm{kDa}$ protein composed of two subunits of $40 \mathrm{kDa}$ : $\alpha$ and $\beta$-chains), and nCLU (encoded $50 \mathrm{kDa}$ ) [5]. sCLU acts as an extracellular chaperone for multiple protein ligands [24,25]. sCLU and psCLU have been suggested to play cytoprotective, anti-inflammatory roles in various organs $[26,27]$. On the other hand, nCLU, a product of an alternative splicing of pre-mRNA, is correlated with the apoptotic response of cells to certain stimuli. [27]. Thus, CLU is known to act a different function in various organs depending on type of isoform and acting location. Furthermore, recent studies have been reported that CLU is closely associated with the inflammation and immune response in human airway: CLU is strongly expressed in the airway submucosa of smokers, and sputum and serum CLU concentration is significantly elevated in patients with severe asthma. CLU modulates the recruitment of inflammatory dendritic cells in the airway [8-10]. In current study, we first identified that the sCLU and nCLU constitutively found in human nasal epithelium. In addition, the expression level of the nCLU is higher than that of sCLU and the level of nCLU of CRSwNP group was higher than that of healthy control group. On the other hand, there was no difference in the expression levels of sCLU between the two groups. These results suggest that nCLU may play an important role in the pathophysiological changes of the nasal epithelium of CRSwNP patients. In addition, the results of our in vitro study show that recombinant nCLU increased MUC5AC expression in NCI-H292 cells and primary culture of human normal nasal epithelial cells. These results suggest a possible association of nCLU with mucus hypersecretion and goblet cell metaplasia in human airway epithelial cells.

To identify the intracellular regulatory mechanism involved in nCLU-induced the expression of MUC5AC, NF- $\mathrm{kB}$ activated was evaluated, because many studies have reported that MUC5AC glycoprotein is increased by the inflammatory mediators which activate the inflammatory transcription factors including NF- $\mathrm{KB}$ and active protein 1 [28,29]. MUC5AC expression is reduced by 6-mercaptopurine via suppression of the NF- $\mathrm{kB}$ path- 
way in airway epithelial cells [30]. Meanwhile, in studies of the association of CLU with NF- $\mathrm{BB}, \mathrm{NF}-\kappa \mathrm{B}$ activation is involved in CLU-induced matrix metalloproteinase-9 expression in monocytes and macrophages [21]. And CLU enhances NF- $\mathrm{BB}$ activation in hyperproliferative synoviocytes of patients with rheumatoid arthritis [31]. Findings of the current study demonstrated that recombinant nCLU increased MUC5AC expression, and significantly activated phosphorylation of NF- $\mathrm{B}$. And BAY 117085 (a specific NF- $\kappa$ B inhibitor) and knockdown of NF- $\kappa B$ by NF- $\kappa \mathrm{B}$ siRNA significantly attenuated recombinant nCLU-induced MUC5AC expression. These results suggest that the activation of NF- $\kappa \mathrm{B}$ is play an important role in nCLU-induced the expression of MUC5AC in human airway epithelial cells.

However, there are some limitations in this study. The present study did not evaluate the changes of the expression of other types of airway mucin genes such as MUC2, 4, 5B, 8, and 16. And the effect of nCLU on the expression of MUC5AC was only checked in two types of submerged culture cells but not in other culture cells grown in different method including air-liquid interface cultures or ex vivo cultures. Also, there was a lack of evidence for the results of this study because the animal study were not performed. Therefore, additional experiments are needed to clarify the results of present study and we are planning further experiments.

In conclusion, the results of this study demonstrate for the first time that CLU was presented in NP and nCLU induced MUC5AC expression via the activation of NF- $\kappa B$ in human airway epithelial cells. Although the effects and the precise signaling pathways of nCLU on the expression of mucin genes in airway epithelial cells remain to be determined, these results provide evidence that CLU may be a key molecule in the development of NP and the regulation of mucus secretion in human airway epithelial cells.

\section{CONFLICT OF INTEREST}

No potential conflict of interest relevant to this article was reported.

\section{ACKNOWLEDGMENTS}

This work was supported by the 2015 Yeungnam University Research Grant, Daegu, Korea.

\section{REFERENCES}

1. Pawankar R, Nonaka M. Inflammatory mechanisms and remodeling in chronic rhinosinusitis and nasal polyps. Curr Allergy Asthma Rep. 2007 Jun;7(3):202-8.
2. Kim HK, Kook JH, Kang KR, Oh DJ, Kim TH, Lee SH. Increased expression of hCLCA1 in chronic rhinosinusitis and its contribution to produce MUC5AC. Laryngoscope. 2016 Nov;126(11):E347-55.

3. Ha EV, Rogers DF. Novel therapies to inhibit mucus synthesis and secretion in airway hypersecretory diseases. Pharmacology. 2016 Jan;97(1-2):84-100.

4. Voynow JA, Rubin BK. Mucins, mucus, and sputum. Chest. 2009 Feb;135(2):505-12.

5. Jeong S, Ledee DR, Gordon GM, Itakura T, Patel N, Martin A, et al. Interaction of clusterin and matrix metalloproteinase- 9 and its implication for epithelial homeostasis and inflammation. Am J Pathol. 2012 May;180(5):2028-39.

6. Park S, Mathis KW, Lee IK. The physiological roles of apolipoprotein $\mathrm{J} /$ clusterin in metabolic and cardiovascular diseases. Rev Endocr Metab Disord. 2014 Mar;15(1):45-53.

7. Sol IS, Kim YH, Park YA, Lee KE, Hong JY, Kim MN, et al. Relationship between sputum clusterin levels and childhood asthma. Clin Exp Allergy. 2016 May;46(5):688-95.

8. Kwon HS, Kim TB, Lee YS, Jeong SH, Bae YJ, Moon KA, et al. Clusterin expression level correlates with increased oxidative stress in asthmatics. Ann Allergy Asthma Immunol. 2014 Mar;112(3):217-21.

9. Hong GH, Kwon HS, Moon KA, Park SY, Park S, Lee KY, et al. Clusterin modulates allergic airway inflammation by attenuating CCL20mediated dendritic cell recruitment. J Immunol. 2016 Mar;196(5): 2021-30.

10. Carnevali S, Luppi F, D’Arca D, Caporali A, Ruggieri MP,Vettori MV, et al. Clusterin decreases oxidative stress in lung fibroblasts exposed to cigarette smoke. Am J Respir Crit Care Med. 2006 Aug;174(4): 393-9.

11. Reddy KB, Karode MC, Harmony AK, Howe PH. Interaction of transforming growth factor beta receptors with apolipoprotein $\mathrm{J} /$ clusterin. Biochemistry. 1996 Jan;35(1):309-14.

12. Santilli G, Aronow BJ, Sala A. Essential requirement of apolipoprotein $\mathrm{J}$ (clusterin) signaling for IkappaB expression and regulation of NF-kappaB activity. J Biol Chem. 2003 Oct;278(40):38214-9.

13. Mourra N, Scriva A, Mansiaux Y, Gozlan S, Bennis M, Balaton A. Clusterin expression in gastrointestinal neuroendocrine tumours is highly correlated with location and is helpful in determining the origin of liver metastases. Histopathology. 2014 Nov;65(5):642-50.

14. Tschopp J, Chonn A, Hertig S, French LE. Clusterin, the human apolipoprotein and complement inhibitor, binds to complement C7, C8 beta, and the b domain of C9. J Immunol. 1993 Aug;151(4):2159-65.

15. Song SY, Woo HJ, Bae CH, Kim YW, Kim YD. Expression of leptin receptor in nasal polyps: leptin as a mucosecretagogue. Laryngoscope. 2010 May;120(5):1046-50.

16. Na HG, Bae CH, Choi YS, Song SY, Kim YD. Spleen tyrosine kinase induces MUC5AC expression in human airway epithelial cell. Am J Rhinol Allergy. 2016 Mar-Apr;30(2):89-93.

17. Kim DH, Chu HS, Lee JY, Hwang SJ, Lee SH, Lee HM. Up-regulation of MUC5AC and MUC5B mucin genes in chronic rhinosinusitis. Arch Otolaryngol Head Neck Surg. 2004 Jun;130(6):747-52.

18. Bae CH, Choi YS, Song SY, Kim YD. Effect of thymic stromal lymphopoietin on MUC5B expression in human airway epithelial cells. Biochem Biophys Res Commun. 2014 May;448(2):231-5.

19. Turner J, Jones CE. Regulation of mucin expression in respiratory diseases. Biochem Soc Trans. 2009 Aug;37(Pt 4):877-81.

20. Woo HJ, Yoo WJ, Bae CH, Song SY, Kim YW, Park SY, et al. Leptin up-regulates MUC5B expression in human airway epithelial cells via mitogen-activated protein kinase pathway. Exp Lung Res. 2010 Jun;36(5):262-9.

21. Essabbani A, Margottin-Goguet F, Chiocchia G. Identification of clusterin domain involved in NF-kappaB pathway regulation. J Biol Chem. 2010 Feb;285(7):4273-7.

22. Pucci S, Bonanno E, Pichiorri F, Angeloni C, Spagnoli LG. Modula- 
tion of different clusterin isoforms in human colon tumorigenesis. Oncogene. 2004 Mar;23(13):2298-304.

23. Bhutia SK, Das SK, Kegelman TP,Azab B, Dash R, Su ZZ, et al. mda7/IL-24 differentially regulates soluble and nuclear clusterin in prostate cancer. J Cell Physiol. 2012 May;227(5):1805-13.

24. Jones SE, Jomary C. Clusterin. Int J Biochem Cell Biol. 2002 May;34(5):427-31.

25.Trougakos IP, Djeu JY, Gonos ES, Boothman DA.Advances and challenges in basic and translational research on clusterin. Cancer Res. 2009 Jan;69(2):403-6.

26. Falgarone G, Chiocchia G. Chapter 8. Clusterin: a multifacet protein at the crossroad of inflammation and autoimmunity. Adv Cancer Res. 2009;104:139-70.

27. Leskov KS, Klokov DY, Li J, Kinsella TJ, Boothman DA. Synthesis and functional analyses of nuclear clusterin, a cell death protein. J Biol Chem. 2003 Mar;278(13):11590-600.
28. Garvin LM, Chen Y, Damsker JM, Rose MC. A novel dissociative steroid VBP15 reduces MUC5AC gene expression in airway epithelial cells but lacks the GRE mediated transcriptional properties of dexamethasone. Pulm Pharmacol Ther. 2016 Jun;38:17-26.

29. Fujisawa T, Velichko S, Thai P, Hung LY, Huang F, Wu R. Regulation of airway MUC5AC expression by IL-1beta and IL-17A; the NFkappaB paradigm. J Immunol. 2009 Nov;183(10):6236-43.

30. Kurakula K, Hamers AA, van Loenen P, de Vries CJ. 6-Mercaptopurine reduces cytokine and Muc5ac expression involving inhibition of NFkB activation in airway epithelial cells. Respir Res. 2015 Jun;16:73.

31. Shim YJ, Kang BH, Jeon HS, Park IS, Lee KU, Lee IK, et al. Clusterin induces matrix metalloproteinase-9 expression via ERK1/2 and PI3K/Akt/NF-kB pathways in monocytes/macrophages. J Leukoc Biol. 2011 Oct;90(4):761-9 